\title{
The Relationship Between Occupational Stress and Health Consequences Among the Workers in Regards with the Perceived Organizational Support
}

\author{
Fariba Kiani $^{*}$, Tayebe Rahimi Pordanjani², Ali Mohamadzadeh Ebrahimi²
}

1. Assistant Professor, Department of Psychology, Boroujen Branch, Islamic Azad University, Boroujen, Iran

2. Assistant Professor, Department of Psychology, Faculty of Humanities, University of Bojnord, Bojnord, Iran

\begin{tabular}{ll}
\hline \multicolumn{2}{c}{ Article Info } \\
Original Article \\
Received: & 11 May 2018 \\
Accepted: & 12 Aug 2018 \\
Published Online & 12 Aug 2018
\end{tabular}

DOI: 10.30699 /jergon.6.1.50

Use your device to scan and read the article online

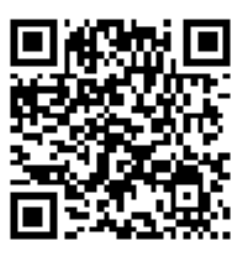

\section{Corresponding Information}

\section{Fariba kiani}

Department of Psychology, Boroujen Branch, Islamic Azad University, Boroujen, Iran

E-mail:

fariba.kiani64@gmail.com

\section{Abstract}

Background: The majority of research have led to interventions such as stress management design and have neglected the role of psychosocial factors in occupational stress. The aim of current research was to the relationship between occupational stress and health consequences among the workers in regards with the perceived organizational support.

Methods: 211 individuals of Isfahan Steel Company were selected according to the stratified random sampling method and completed questionnaires about demography characteristics, Occupational stress, incident reporting rate and perceived organizational support. The collected data were analyzed by descriptive index and multiple regression analysis using SPSS18.

Results: Results showed that there were significant relationships among perceived organizational support, Occupational stress and incident reporting rate $(P<0.05)$. Also, hierarchy regression analysis showed that the relationship between Occupational stress and incident reporting rate was partially mediated by perceived organizational support $(P<0.05)$.

Conclusion: The results of this study indicate that perceived organizational support can modify the relationship between occupational stress and involvement in work accidents, and as a result, employees are less involved in risk behaviors and accidents are less caused by occupational stress.

Keywords: Social Support, Occupational stress, Accidents

Copyright (C) 2018, Journal of Ergonomics. This is an open-access article distributed under the terms of the Creative Commons Attribution-noncommercial 4.0 International License which permits copy and redistribute the material just in noncommercial usages, provided the original work is properly cited

How to Cite This Article:

Kiani F, Rahimi Pordanjani T, Mohamadzadeh Ebrahimi A. The Relationship Between Occupational Stress and Health Consequences Among the Workers in Regards with the Perceived Organizational Support. J Ergon. 2018; 6 (1): 50-57 


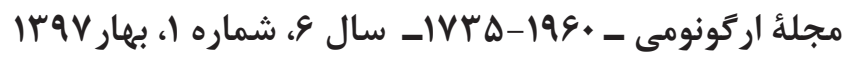

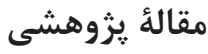

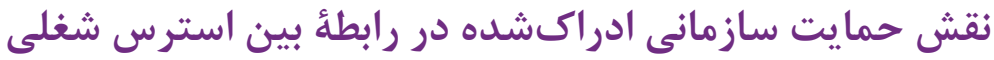 \\ با بيامدهاى سلامت در كاركنان
}

فريبا كيانى'"'، طيبه رحيمى يردنجانى '، على محمدز اده ابر اهيمى'

I. استاديار، تروه روانشناسى، واحد بروجن، دانشغاه آزاد اسلامى، بروجن، ايران

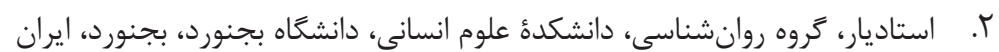

\begin{abstract}
جكيده
زمينه و هدف: غالب يزوهشهاى انجامشده پِيرامون استرس شغلى بر طراحى مداخلاتى مثل مديريت

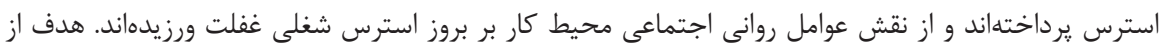

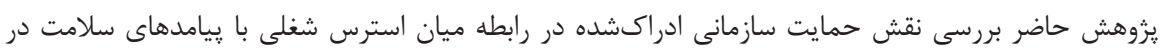

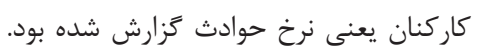

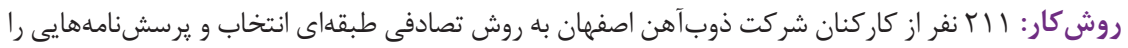

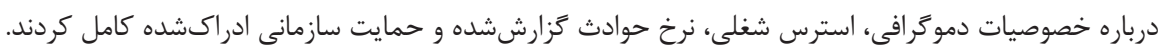

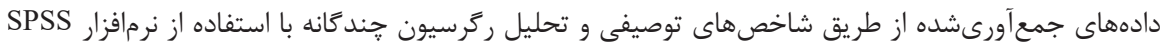

نسخة \1 ا تجزيهوتحليل شدند.

يافتهها: نتايج نشان داد كه بين متغيرهاى حمايت سازمانى ادراكشده، استرش شغلى و نرخ حوادث

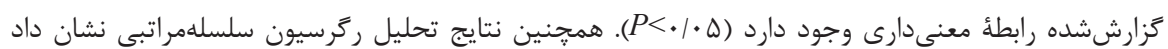

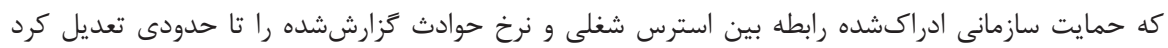

$$
(P<\cdot / \cdot \bowtie)
$$

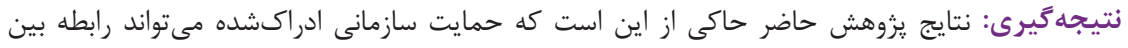

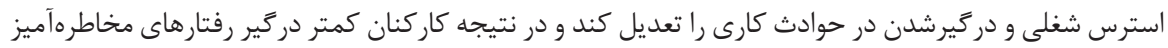

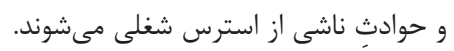

وازههاى كليدى: حمايت اجتماعى، استرس شغلى، حوادث
\end{abstract}

مقابله است [ع]. عوامل زيادى در محيط كار وجود دارد كه

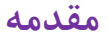

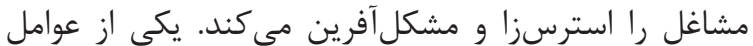

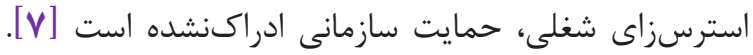

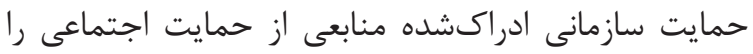

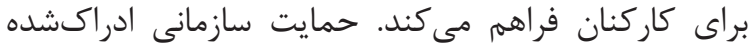

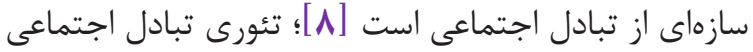

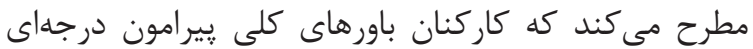

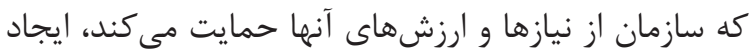

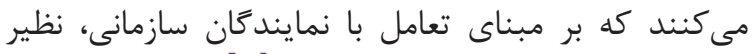

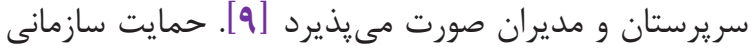

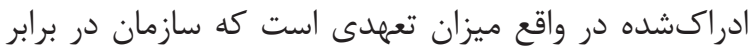

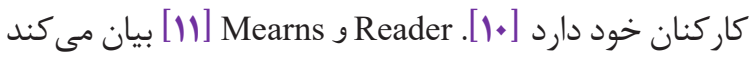

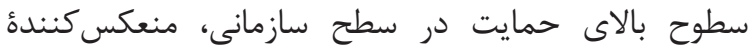

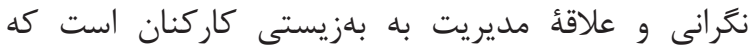
منجر به روابط متقابل و افزايش رفتار شهروندى ايمنى (Safety Citizenship Behavior) ديخر، تبادل اجتماعى درون يك سازمان منجر به فيه فوايد

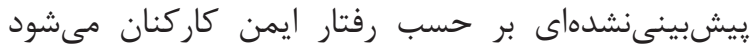

اطلاعات مقاله - اله

\begin{tabular}{|c|c|}
\hline$|r q V / \cdot r / T|$ & ول: \\
\hline$|r q \nabla / \cdot \Delta / r|$ & ناريخ ؛ \\
\hline$|r q v / \cdot \Delta / r|$ & 1 \\
\hline
\end{tabular}

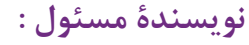
فريبا كيانى

كروه روانشناسى، واحد بروجن،

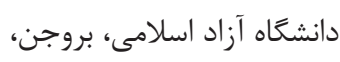

يست الكترونيك:

fariba.kiani64@gmail.com 


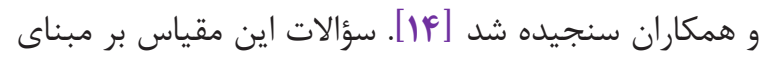

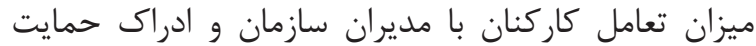

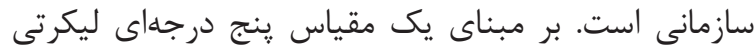

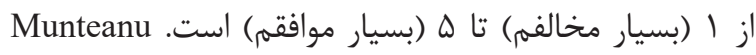

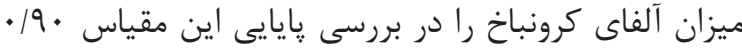

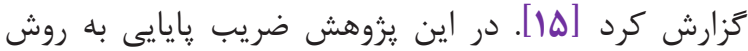

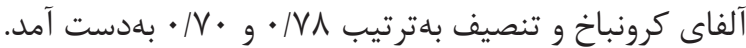

نرخ حوادث كزارششده با أب آيتم از Barling و همكاران

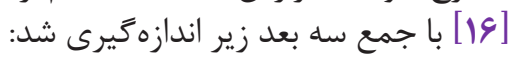

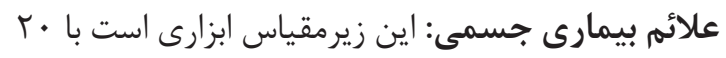

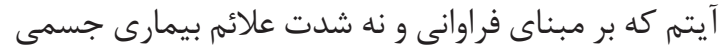

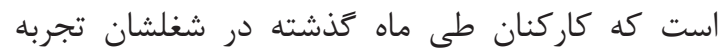

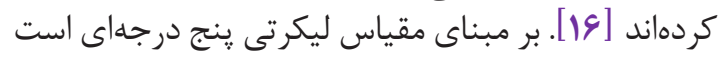

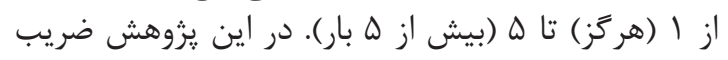

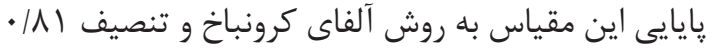

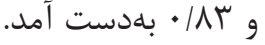

علائم بيمارى روانشناختى: اين زيرمقياس ابزارى است

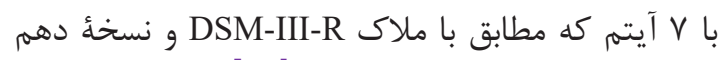

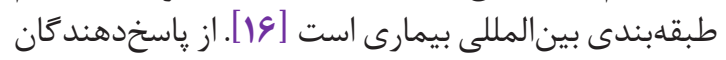

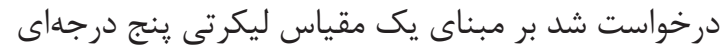

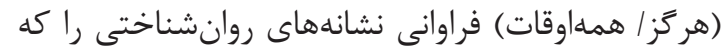

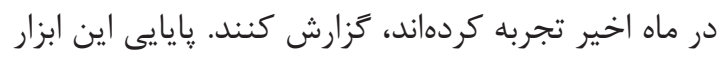

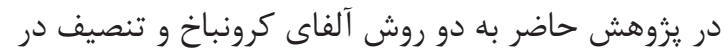

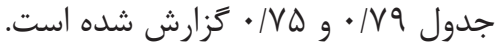

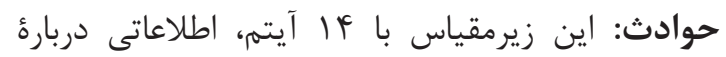

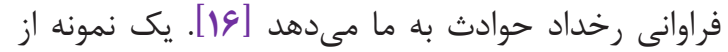

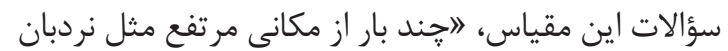

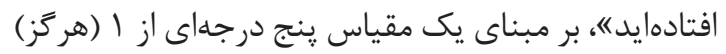

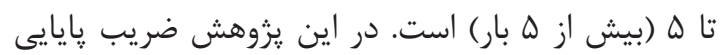

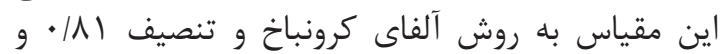

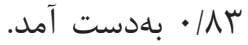

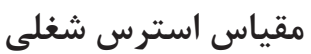

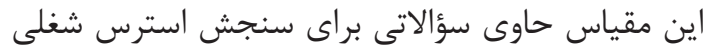

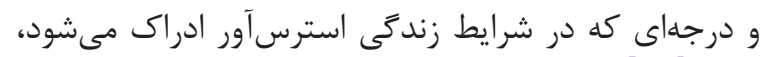

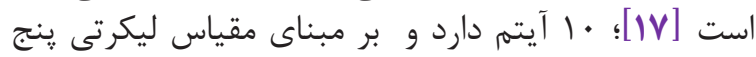

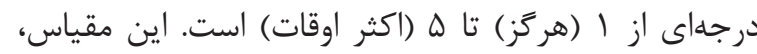

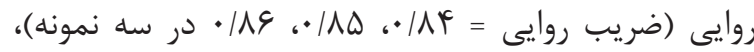

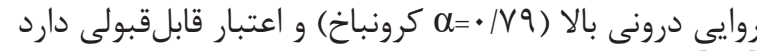

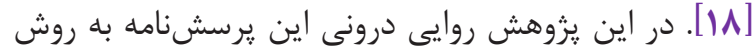

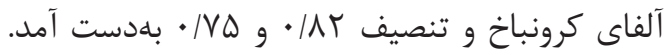

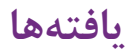

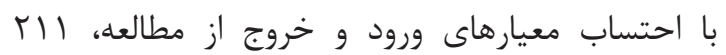

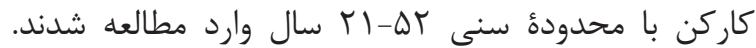

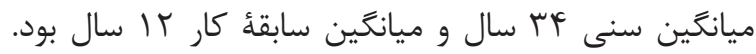

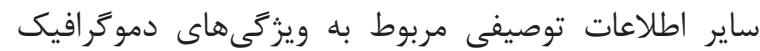

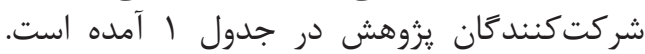

كه فراتر از متابعت بهنجار است. حمايت سازمان و بهويزه دانيه

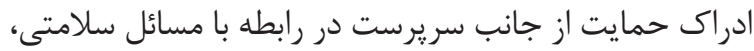

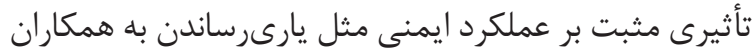

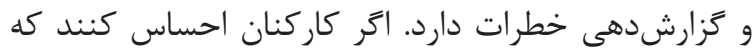

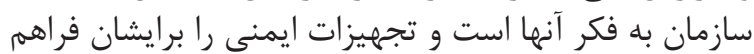

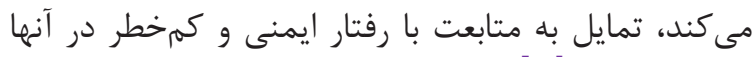

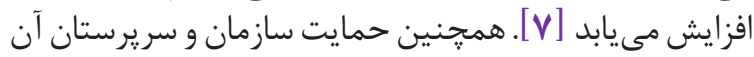

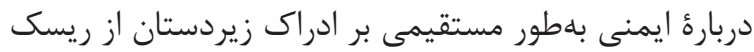

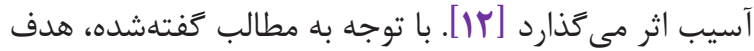

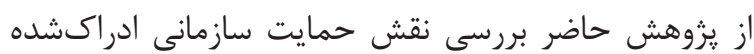

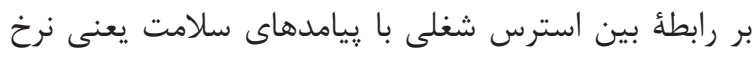
حوادث گزارششده است.

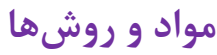

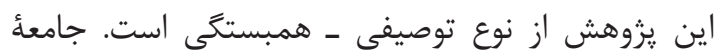

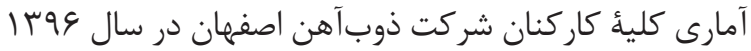

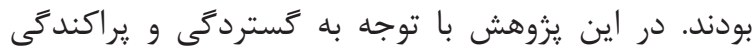

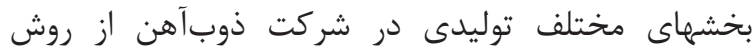

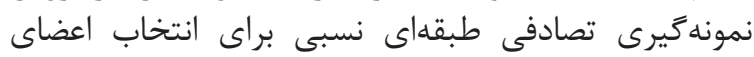

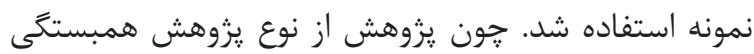

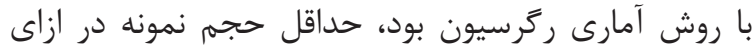

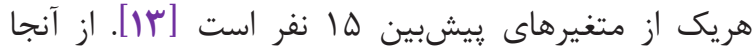

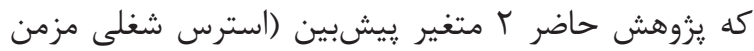

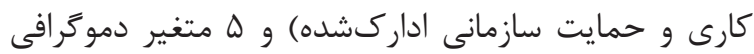

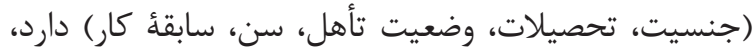

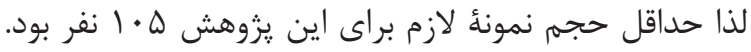

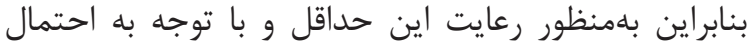

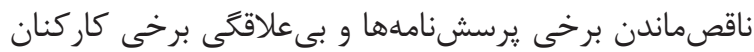

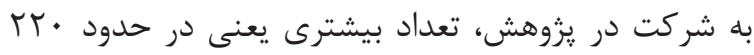

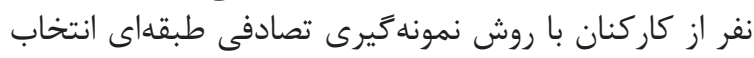

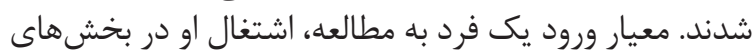

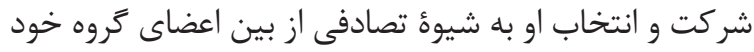

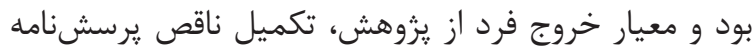

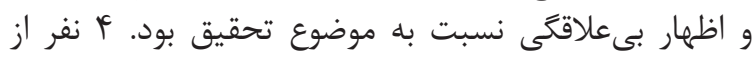

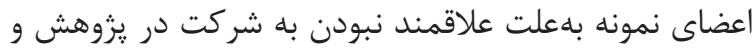

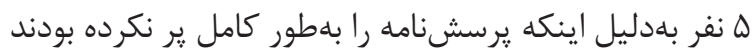

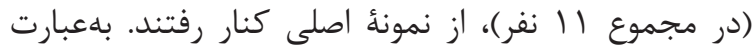

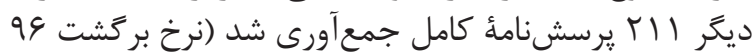

درص).

بهمنظور كنترل عوامل مخدوش كنتنده، اعضاى نمونه

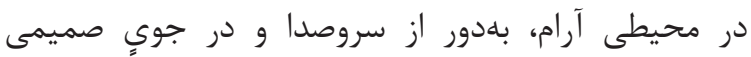

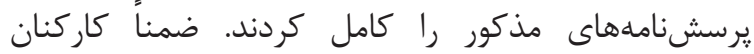

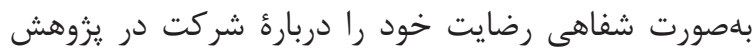

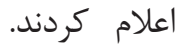

بهمنظور جمعآورى اطلاعات از مقياسهاى زير استفاده شد:

\section{مقياس حمايت سازمانى ادراكشده براى ايمنى}

حمايت سازمانى ادراكشده با r T آيته از مقياست 


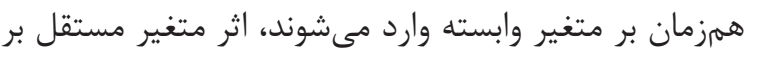

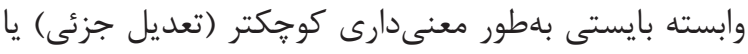
ديخر معنى دار نباشد (تعديل كامل). از نرمافزار SPSS نسخئه

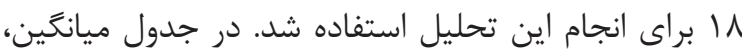
انحراف استاندارد، همبستخى درونى درونى بين متغيرها ارائه شده است.

همانطور كه در جدول r مشاهده مىشود، بين متغيرهاى

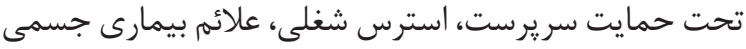
و روانشناختى همبستخى بالايى وجود دارد (ه •|•>P).

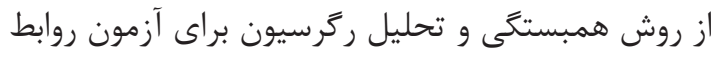

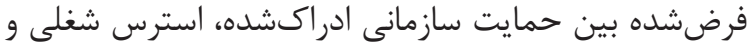

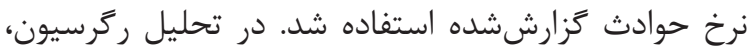

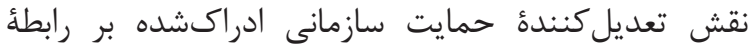

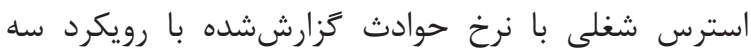

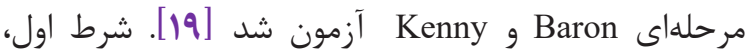

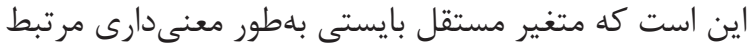

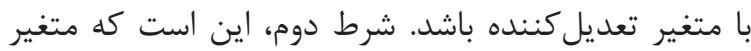

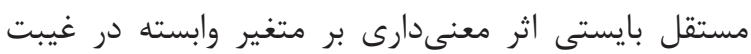

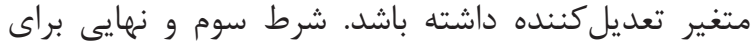

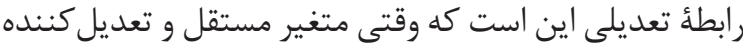

جدول ا. ويرَّى هاى دموكَرافى شركت كنندكًان يُوهش (N = rII)

\begin{tabular}{|c|c|c|c|}
\hline درصد & فراوانى & ،موتر افى & ويز \\
\hline$\% 4$ & VQ & 11 تا 9r سال & \multirow{3}{*}{ سن } \\
\hline$\% r_{4}$ & $V \Delta$ & •r تا أf سال & \\
\hline$\% \curlyvee \wedge$ & 91 & rF تا Fه سال & \\
\hline$\% 1$ & rI & 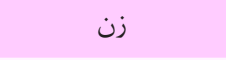 & \multirow[b]{2}{*}{ جنسيت } \\
\hline$\% 9$. & 19. & 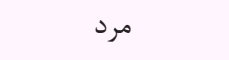 & \\
\hline$\%$ & NF & مجرد & \multirow{2}{*}{ وضعيت تأهل } \\
\hline$\% 9$. & ITV & متأهل & \\
\hline$\%$ & $\wedge$ & زير دييلم & \multirow{4}{*}{ تحصيلات } \\
\hline$\%$ & Irs & دييلم & \\
\hline$\% Y r$ & $\Delta \cdot$ & فوق دييلم & \\
\hline$\%$ & TV & ليسانس و بالاتر & \\
\hline$\%$ & $V \Delta$ & ه سال و يايينتر & \multirow{4}{*}{ سابقهُ كار } \\
\hline$\% r$ & $\Delta$. & צ تا ها سال & \\
\hline$\%$ Yr & $\Delta \cdot$ & عا تا T سال & \\
\hline$\% 19$ & re & צr سال و بالاتر & \\
\hline \% & $r \Delta$ & كارمند و كارشناس & \multirow{3}{*}{ سمت } \\
\hline$\% \wedge \wedge$ & 119 & ايراتور و تعمير كار & \\
\hline- & - & مدير & \\
\hline$\% r_{4}$ & VD & روزكار & \multirow{2}{*}{ نوع شيفت } \\
\hline$\% 94$ & 1re & شيفت كار & \\
\hline
\end{tabular}

جدول r. ميانغَين، انحر اف استاندارد، همبستگى درونى متغيرها (N=119)

\begin{tabular}{|c|c|c|c|c|c|}
\hline \multicolumn{5}{|c|}{ ضرايب همبستَّى } & \\
\hline \multirow[t]{3}{*}{ r } & r & 1 & SD & $X$ & \\
\hline & & 1 & $4 / 94$ & $r \Delta / r \Delta$ & استرس شغلى \\
\hline & 1 & $* * / r y$ & $\Delta / \cdot r$ & $19 / 94$ & نرخ حوادث تزارش شده \\
\hline 1 & ***-• & $*-\cdot / r \Delta$ & $r / \Delta \mid$ & $r q / 1$ & حمايت سازمانى ادراكشده \\
\hline
\end{tabular}


در كام دوم، حمايت سازمانى ادراكشده پِيشبين معنى دارِ

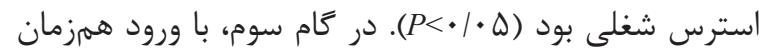

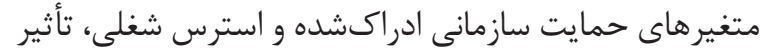

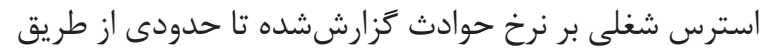

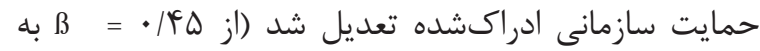

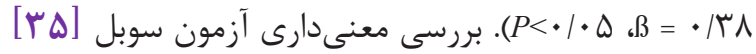

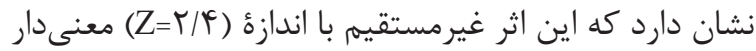

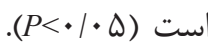

در جدول با، نتايج تحليل ركرسيون تعديلى ارائه شده

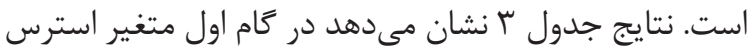

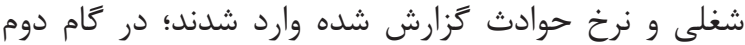

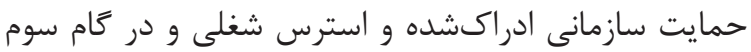

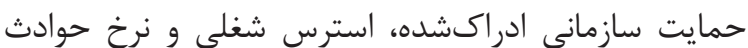

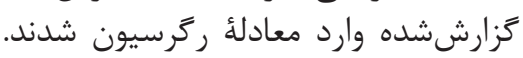

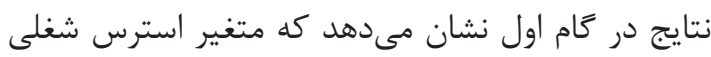

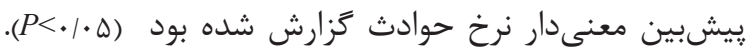

جدول ץ. نتايج تحليل ركرسيون تعديلى

\begin{tabular}{|c|c|c|c|c|c|}
\hline Sig & $\mathbf{T}$ & B & SE & B & كامهاى بارون و كنى (1919) \\
\hline \multicolumn{6}{|c|}{ اثرات مستقيم و كلى } \\
\hline$\cdot 1 \cdots$ & $F / F i$ & $\cdot / \& \Delta$ & $\cdot 1 \cdot \mathrm{Vq}$ & $\cdot / \pi \Delta$ & 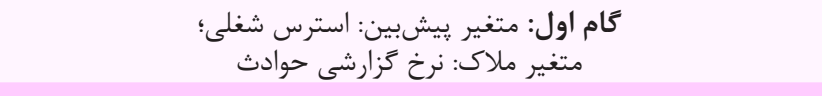 \\
\hline$\cdot / \cdot r$ & $-r / r q$ & $-\cdot / r 4$ & $\cdot 1 \cdot 49$ &.$- / 14$ & كام دوم: متغير يِشبين: حمايت سازمانى استى ادراكشده؛ \\
\hline . & $-r / 19$ & $\cdot / \mu \wedge$ & $\cdot / \cdot 1$ & $\cdot / r \cdot$ & 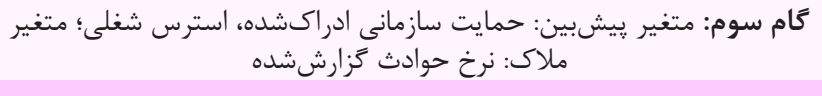 \\
\hline \multicolumn{6}{|c|}{ اثر غيرمستقيم و معنىدارى با استفاده از توزيع } \\
\hline $\mathrm{P}$ & Z & & & & \multirow{2}{*}{ 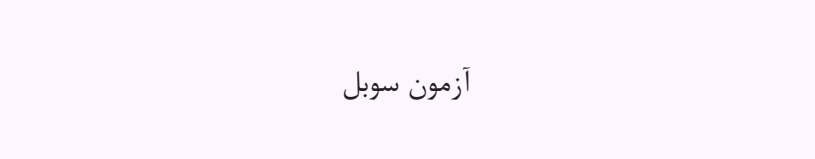 } \\
\hline . $/ . r$ & $r / F$ & & & & \\
\hline
\end{tabular}

ييشينهُ يزوهشى موجود، از اين ادعا كه حمايت سازمانى

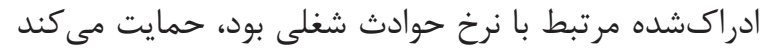

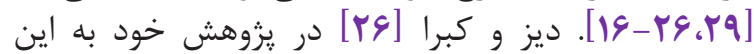

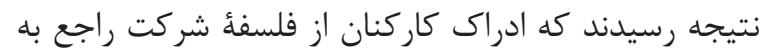

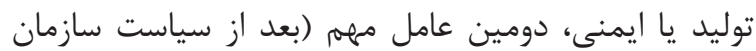

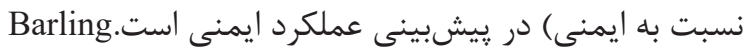

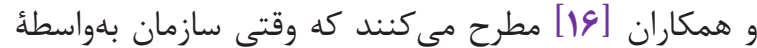

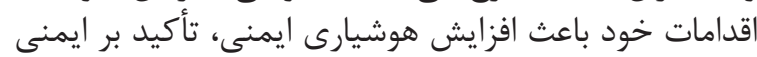

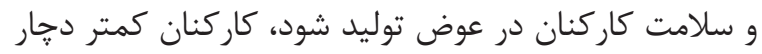

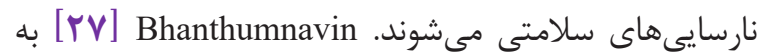

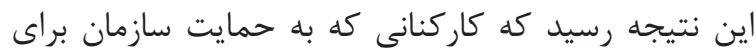

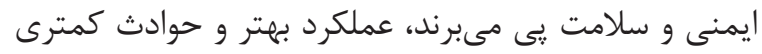

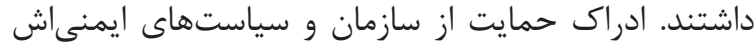

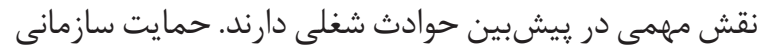

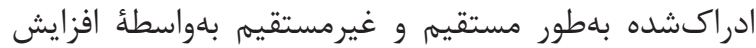

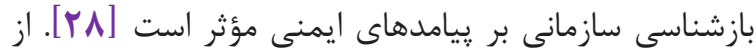

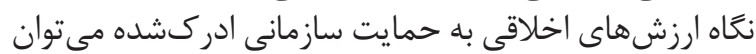

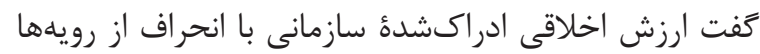

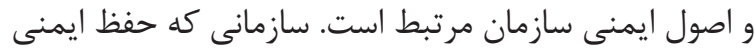

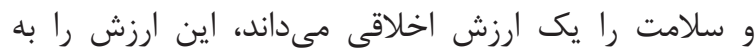

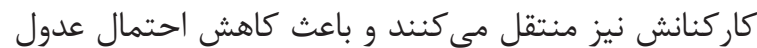

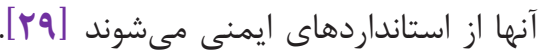

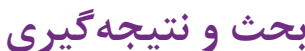

هدف از يزوهش حاضر بررسى نقش حمايت سازمانى

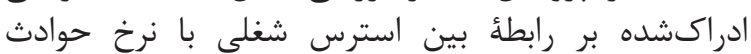

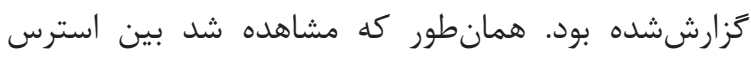

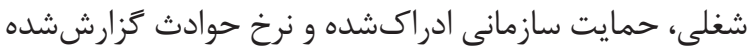

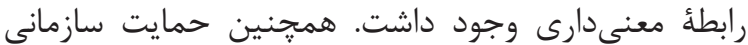

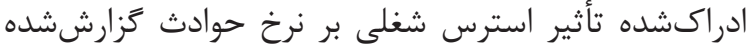
را تا حدودى تعديل كرد.

يشيشئه يزوهشى از اين ادعا كه حمايت سازمانى

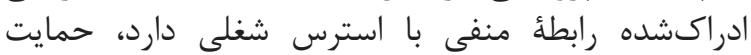

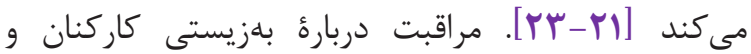

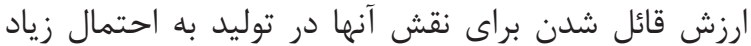

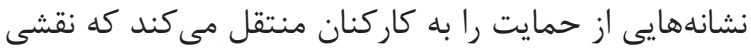

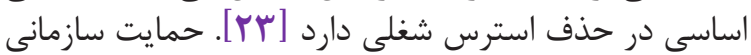

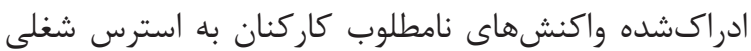

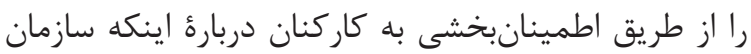

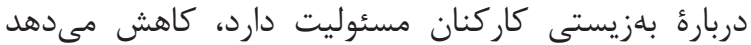

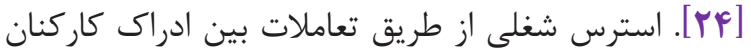

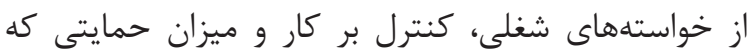

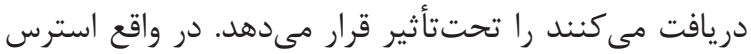

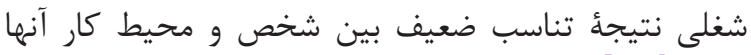

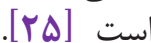


بين استرس و حوادث است. نقش ضربهَّير استرس از فرد در

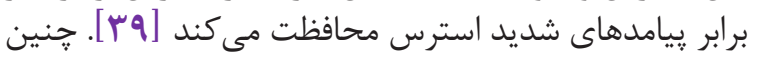

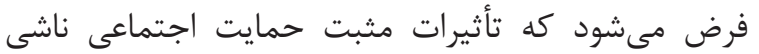

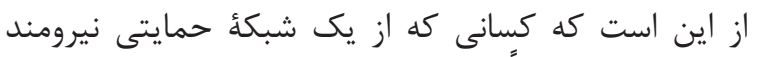

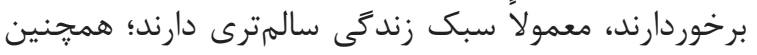

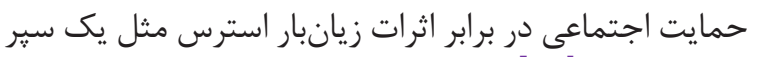

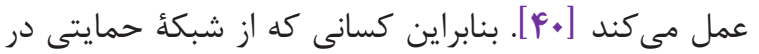

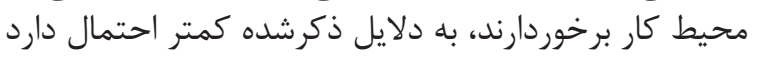

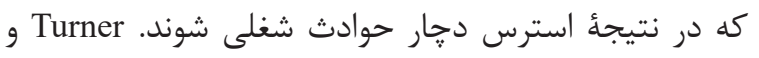

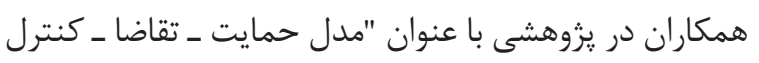

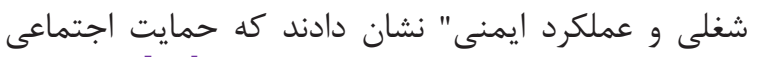

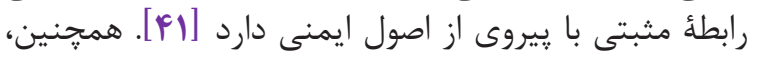

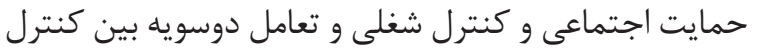

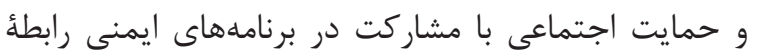

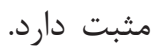

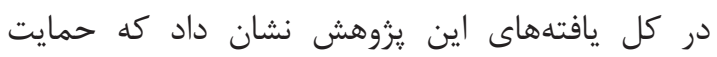

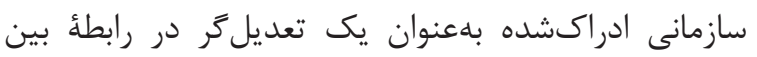

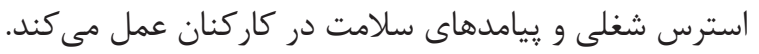

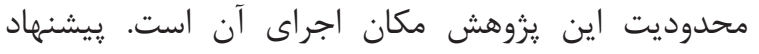

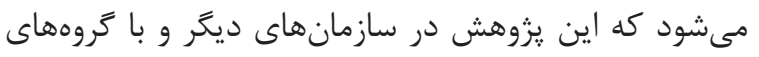

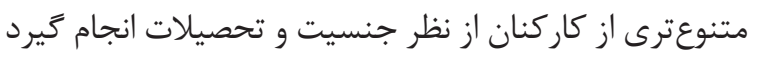

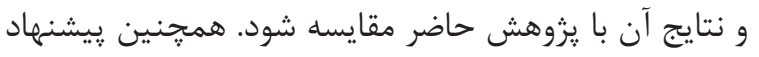

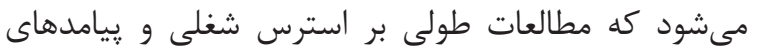

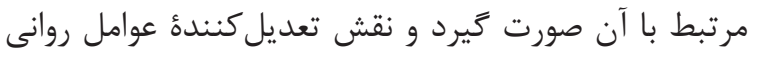

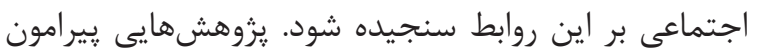

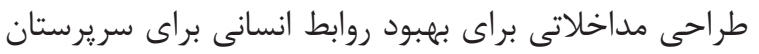

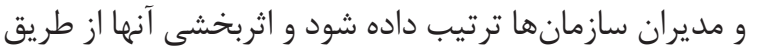

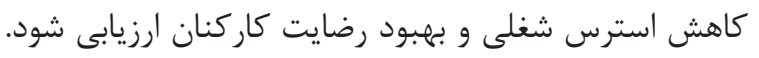

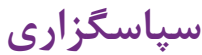

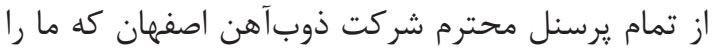

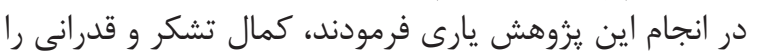
داريم.

\section{تعارض منافع}

بين نويسندكان هيجَّونه تعارضى در منافع وجود ندارد.

\section{References}

1. Rehman SU, Khan MA, Afzal H, Akhter W, Imran A. Stress in banker's life: Demands-control model as predictors of employee's activity participation. Afr. J. Bus. Manage. 2010;4(9):1679-90.

2. Mcfarlane AC. The long-term costs of traumatic stress: intertwined physical and psychological consequences. World Psychiatry. 2010;9(1):3-10. PMID:20148146 PMCID:PMC2816923

3. Smith TD, Hughes K, DeJoy DM, Dyal MA. Assessment of relationships between work stress, work-family conflict, burnout and firefight-
دربارهٔ اثر تعديل كننده حمايت سازمانى ادراكشده بر

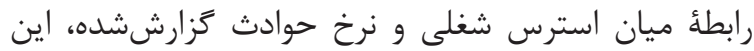

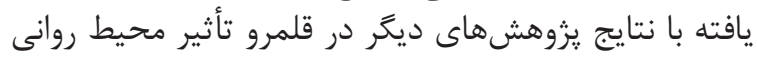

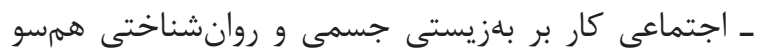

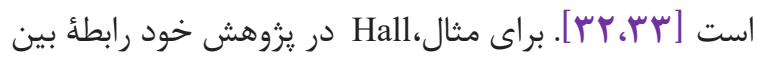

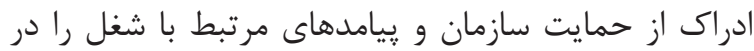

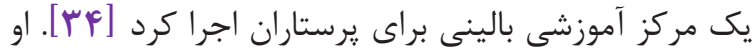

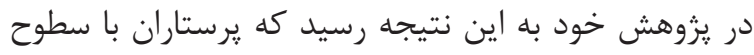

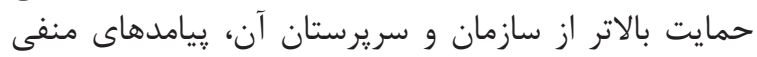

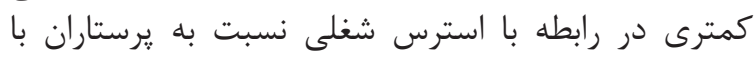

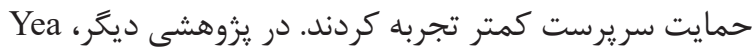

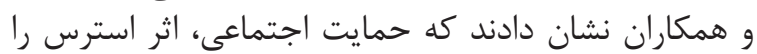

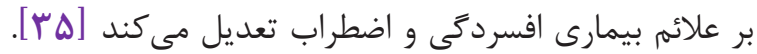

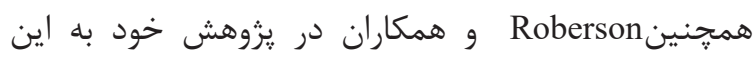

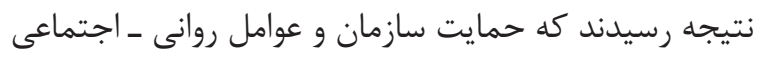

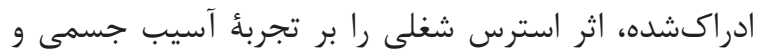

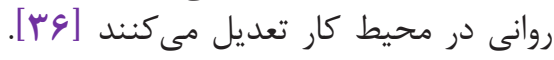

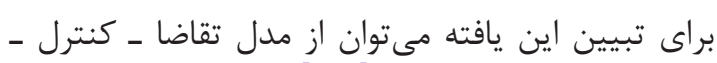

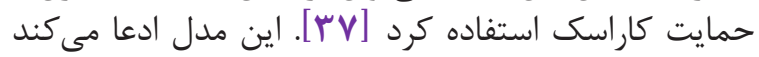

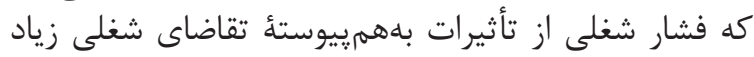

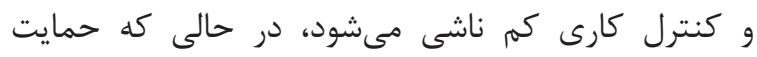

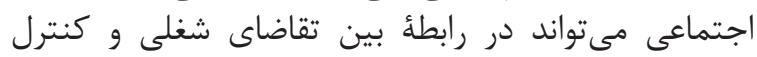

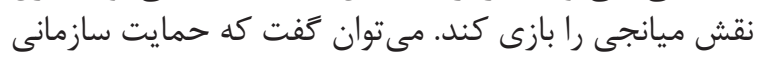

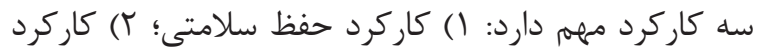

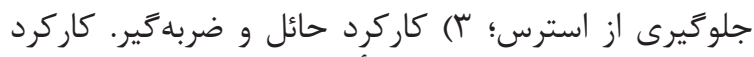

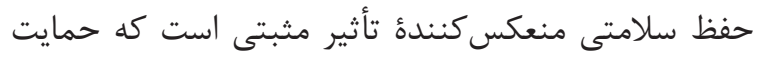

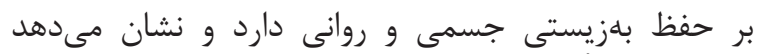

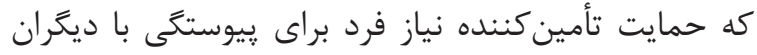

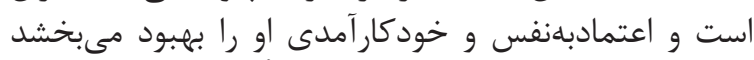

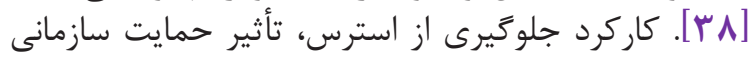

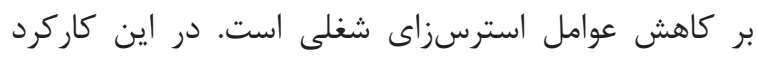

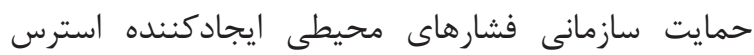
را كاهش مى ندهد و در اين زمينه شواهدى فئى وجود دارئ دارد كه

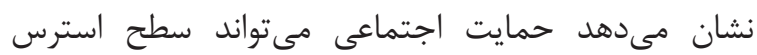

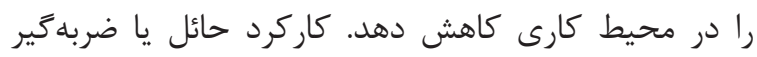

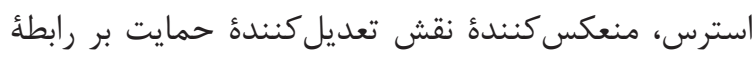

er safety behavior outcomes. Safety Science. 2018;103:287-92. $\quad$ https://doi.org/10.1016/j. $\underline{\text { ssci.2017.12.005 }}$

4. Junne F, Michaelis M, Rothermund E, Stuber F, Gündel H, Zipfel S, Rieger MA. The Role of Work-Related Factors in the Development of Psychological Distress and Associated Mental Disorders: Differential Views of Human Resource Managers, Occupational Physicians, Primary Care Physicians and Psychotherapists in Germany. Int J Environ Res Public Health. 2018;15(3):559. https://dx.doi.org/10.3390\%2Fijerph15030559 PMID:29558427 PMCID:PMC5877104 
5. Khamisa N, Peltzer K, Ilic D, Oldenburg B. Effect of personal and work stress on burnout, job satisfaction and general health of hospital nurses in South Africa. health sa gesondheid 2017;22(1):252-8. https://doi.org/10.1016/j. hsag.2016.10.001

6. Levi L. Spice of life or kiss of death? In C. L. Cooper (Ed.). Handbook of Stress, Medicine and Health, Boca Raton, Fl: CRC Press 1996; 1-10.

7. Reader TW, Mearns K, Lopes C, Kuha J. Organizational support for the workforce and employee safety citizenship behaviors: A social exchange relationship. Human Relations. 2017;70(3):36285. https://doi.org/10.1177/0018726716655863

8. Kurtessis JN, Eisenberger R, Ford MT, Buffardi LC, Stewart KA, Adis CS. Perceived organizational support: A meta-analytic evaluation of organizational support theory. Journal of Management. 2017;43(6):1854-84. https://doi. org/10.1177/0149206315575554

9. Rhoades L, Eisenberger R. Perceived organizational support: A review of the literature. J Appl Psychol. 2002;87:698-714. https://doi.org/10.1037/0021-9010.87.4.698 PMID: 12184574

10. Leenu N, Lakhwinder S. Role of Perceived Organizational Support in the Relationship between HR practices and organizational trust. Global Business Review. 2012;13(2):239-49. https://doi. org/10.1177/097215091201300204

11. Mearns KL, Reader, T. Organizational support and safety outcomes: An un-investigated relationship? Safety Science. 2008;46(3):388-97. https://doi.org/10.1016/j.ssci.2007.05.002

12. Wong KC. Work support, psychological well-being and safety performance among nurses in Hong Kong. Psychol Health Med. 2018;23(8):958-63. https://doi.org/10.1080/13548506.2018.1437275 PMID:29405069

13. Frahani H, Oreyzi HR. Advanced methods in human sciences. 2nd ed. Isfahan: University Jehad publications; 2005.

14. Hayes BE, Perander J, Smecko T, Trask J. Measuring perceptions of workplace safety: development and validation of the work safety scale. Journal of Safety Research. 1998;29(3):145-61. https://doi.org/10.1016/S0022-4375(98)00011-5

15. Munteanu MR. Safety attitudes in the Ontario construction. University of Toronto; 2005. p.1165.

16. Barling J, Loughlin C, Kelloway EK. Development and test of a model linking safety-specific transformational leadership and occupational safety. J Appl Psychol. 2002;87(3):488-96. https://doi.org/10.1037/0021-9010.87.3.488 PMID: 12090606

17. Cohen S, Karmark T, Mermelstein RA. Global measure of perceived stress. J Health Soc Behav. 1983;24(4):385-96. https://doi. org/10.2307/2136404 PMID: $\underline{6668417}$

18. Teresa EA. Associations of mindfulness, perceived stress, and health behaviors in college freshmen. Arizona State University; 2008. p.191

19. Baron RM, Kenny DA. The moderator-mediator variable distinction in social psychology research: Conceptual, strategic, and statistical considerations. J Pers Soc Psychol. 1986;51(6):1173-82. https://doi.org/10.1037/0022-3514.51.6.1173 PMID:3806354

20. Preacher KJ, Hayes AF. SPSS and SAS procedures for estimating indirect effects in simple mediation models. Behavior Research Methods, Instruments, and Computers. 2004;36(4):717-31. https://doi.org/10.3758/BF03206553

21. Schaufeli WB, Bakker AB. Occupational demands, Occupational resources, and their relationship with burnout and engagement: A multi-sample study. J Organ Behav. 2004;25(3):293-315. https://doi.org/10.1002/job.248

22. Van den Broeck A, Vansteenkiste M, De Witte $\mathrm{H}$, Lens W. Explaining the relationships between Occupational characteristics, burnout, and engagement: The role of basic psychological need satisfaction. Work and Stress. 2008;22(3):277-94. https://doi.org/10.1080/02678370802393672

23. Törner M, Pousette A, Larsman P, Hemlin S. Coping with paradoxical demands through an organizational climate of perceived organizational support: an empirical study among workers in construction and mining industry. The Journal of Applied Behavioral Science. 2017;53(1):117-41. https://doi.org/10.1177/0021886316671577

24. Giauque, D, Anderfuhren-Biget S, Varone F. Stress Perception in Public Organizations: Expanding the Occupational Demands-Occupational Resources Model by Including Public Service Motivation. Review of Public Personnel Administration. 2013; 33(1):58-83. https://doi. org/10.1177\%2F0734371X12443264

25. Vassos MV, Nankervis KL. Investigating the importance of various individual, interpersonal, organisational and demographic variables when predicting Occupational burnout in disability support workers. Res Dev Disabil. 2012;33(6):178091. https://doi.org/10.1016/j.ridd.2012.04.016 PMID:22699251

26. .Diaz RT, Cabrera DD. Safety climate and attitude as evaluation measures of organizational 
safety. Accid Anal Prev. 1997;29(5):643-50. https://doi.org/10.1016/S0001-4575(97)00015-8 PMID: 9316712

27. Bhanthumnavin D. Perceived social support from supervisor and group members' psychological and situational characteristics as predictors of subordinate performance in Thai work units. Human Resource Development Quarterly. 2003;14(1):79-97. http://psycnet.apa.org/ doi/10.1002/hrdq.1051

28. Edwards MR, Peccei R. Perceived organizational support, organizational identification, and employee outcomes. Pers Psychol. 2012;9(1)17-26. https://doi.org/10.1027/1866-5888/a000007

29. Biron M. Negative reciprocity and the association between perceived organizational ethical values and organizational. Human Relations. 2010;63(6):875-97. https://doi. org/10.1177\%2F0018726709347159

30. Huang YH, Chen PY, Krauss AD, Rogers A. Quality of the execution of corporate safety policies and employee safety outcomes: assessing the moderating role of supervisor safety support and the mediating role of employee safety control. Journal of Business and Psychology. 2004;18(4):483-506. https://link.springer.com/article/10.1023/B:JOBU.0000028448.01394.bf

31. Gray-Stanley JAG, Muramatsu T, Heller T, Hughes S, Johnson T.P, Ramirez-Valles J. Work stress and depression among direct support professional: the role of work support and locus of control. J Intellect Disabil Res. 2010; 54(8):749-61. https://doi.org/10.1111/j.13652788.2010.01303.x PMID:20633203

32. Desouky D, Allam H. Occupational stress, anxiety and depression among Egyptian teachers. Journal of epidemiology and global health. 2017;7(3):191-8.https://doi.org/10.1016/j. jegh.2017.06.002 PMID:28756829

33. Törner M, Pousette A, Larsman P, Hemlin S. Coping with paradoxical demands through an organizational climate of perceived organizational support: an empirical study among workers in construction and mining industry. The Journal of
Applied Behavioral Science. 2017;53(1):117-41. https://doi.org/10.1177/0021886316671577

34. Hall DS. The relationship between supervisor support and registered nurse outcomes in nursing care units. Nurs Adm Q. 2007;31(1):68-80. https://doi.org/10.1097/00006216-20070100000015 PMID:17198122

35. Yea XQ, Chenb WQ, Lina JX, Wanqe RP, Zhange ZH, Yanqe X, Yua XQ. Effect of social support on psychological-stress-induced anxiety and depressive symptoms in patients receiving peritoneal dialysis. J Psychosom Res. 2008;65(2):157-64. https://doi.org/10.1016/j.jpsychores.2008.04.007 PMID: 18655861

36. Roberson M, Hunag E, Larson N. Examining the effects of workstation design satisfaction, computer, computer usage, supervisory and co-worker on perceived physical discomfort and psychological factors. Ergonomics and Health Aspects of Work with Computers. 2009;56(24):88-94. https://doi.org/10.1007/978-3-642-02731-4 11

37. Karasek Jr RA. Job demands, job decision latitude, and mental strain: Implications for job redesign. Administrative Science Quarterly. 1979:285-308. https://doi.org/10.2307/2392498

38. Brouwers A, Evers WJ, Tomic W. Self-efficacy in eliciting social support and burnout among secondary-school teachers. Journal of Applied Social Psychology. 2001;31(7):1474-91. https:// doi.org/10.1111/j.1559-1816.2001.tb02683.x

39. Asnani V, Pandey UD, Sawhney M. Social support and occupational health of working women. Journal of Health Management. 2004;6(2):129-39. https://doi.org/10.1177/097206340400600204

40. André-Petersson L, Engström G, Hedblad B, Janzon L, Rosvall M. Social support at work and the risk of myocardial infarction and stroke in women and men. Soc Sci Med. 2007;64(4):830-41. https://doi.org/10.1016/j.socscimed.2006.10.020

41. Turner N, Stride CB, Carter AJ, McCaughey D, Carroll AE. Job Demands-Control-Support model and employee safety performance. Accid Anal Prev. 2012;45:811-7. https://doi.org/10.1016/j. aap.2011.07.005 PMID:22269573 